\title{
Development and validation of the Adolescent Asthma Quality of Life Questionnaire (AAQOL)
}

\author{
C. Rutishauser*, S.M. Sawyer*,\#, L. Bond*, C. Coffey*, G. Bowes
}

\begin{abstract}
Development and validation of the Adolescent Asthma Quality of Life Questionnaire (AAQOL). C. Rutishauser, S.M. Sawyer, L. Bond, C. Coffey, G. Bowes. C ERS Journals Ltd 2001.

ABSTRACT: Current asthma-specific quality of life questionnaires have major conceptual and methodological deficiencies for use in adolescents. The aim of this study was to develop and validate the "Adolescent Asthma Quality of Life Questionnaire (AAQOL)", specifically developed for adolescents with asthma.

One-hundred and eleven adolescents with frequent-episodic or persistent asthma aged 12-17 yrs were recruited from three tertiary paediatric asthma clinics. The standardized multi-step method consisted of: 1) item selection including semistructured interviews $(n=14) ; 2)$ item reduction and validation $(n=66)$; and 3$)$ assessment of reproducibility $(n=31)$. Item reduction was performed applying the clinical impact method.

The 32 item AAQOL covers six domains: symptoms, medication, physical activities, emotion, social interaction and positive effects. There was high internal consistency for the six domains $(\alpha=0.70-0.90)$ and for the total score $(\alpha=0.93)$. Test-retest reliability was high for all domain scores $(r=0.76-0.85)$ and the total score $(r=0.90)$, indicating high reproducibility of the AAQOL. There was high correlation with the paediatric Asthma Quality of Life Questionnaire $(\rho=0.81)$ which focuses primarily on symptoms and emotional well-being. There was weak to moderate correlation with clinical parameters of asthma severity $(\rho=0.25-0.65)$.
\end{abstract}

The 32-item Adolescent Asthma Quality of Life Questionnaire is a valid, developmentally age-appropriate and dimensionally comprehensive asthma-specific quality of life measure for use in adolescents.

Eur Respir J 2001; 17: 52-58.

\begin{abstract}
*Centre for Adolescent Health, Royal Children's Hospital, "Dept of Respiratory Medicine, Royal Children's Hospital, and The University of Melbourne, Royal Women's and Children's Health Care Network and The University of Melbourne, Melbourne, VIC, Australia.
\end{abstract}

Correspondence: C. Rutishauser, University Children's Hospital, Steinwiesstrasse 75, CH-8032 Zurich, Switzerland. Fax: 4112667171

Keywords: Adolescents

asthma

outcome assessment

quality of life

Received: November 261999

Accepted after revision August 212000

This research was supported by VicHealth (Australia), Asthma Ligue of Zurich, Novartis-Jubilee Foundation, and Theodor and Ida Herzog-Egli Foundation (Switzerland).
Health-related quality of life (HRQOL) is recognized as an important health outcome measure in asthma. In contrast to physiological outcome measures, the aim of HRQOL, instruments is to assess the impact asthma has on a person's daily functioning and emotional wellbeing. Asthma-specific HRQOL instruments have been available for use in adults for several years, but it is only recently that a number of asthma-specific HRQOL instruments have been developed for children and adolescents.

Four asthma-specific HRQOL instruments are currently available to use with children and adolescents [1-4]. These instruments all have major conceptual and/ or methodological deficiencies, particularly for use in adolescents [5]. Adolescence is defined as the transitional period of development from childhood to adulthood [6]. Within this period of transition, immense physical, cognitive, social and emotional changes occur which render adolescents particularly vulnerable to the impact of a chronic illness such as asthma, and suggests that HRQOL research should be separately addressed in adolescents [7]. This is particularly important for disease-specific HRQOL instruments, as these have more specific questions than generic measures. Currently, only the "Childhood Asthma Questionnaire" developed by FRENCH et al. [2] considers these developmental differences by providing different versions for different age groups. Unfortunately, this instrument has other deficiencies which limit its usefulness [5]. The "Pediatric Asthma Quality of Life Questionnaire (PAQLQ)" [1] is designed for the broad age range of 7-17 yrs. Although it includes a domain "limitations in activities", its major focus is on symptoms (10/23 items) and emotional function (8/23). While these domains include items important to any age group, they do not cover the broad spectrum of HRQOL domains of particular relevance to adolescents.

This paper describes the development and validation of a new asthma-specific HRQOL instrument specifically designed for use in adolescents. According to the definition of HRQOL which "represents the functional effect of an illness and its consequent therapy upon a patient, as perceived by the patient" [6], the Adolescent Asthma Quality of Life Questionnaire was developed to fit: 1) target age 12-17 yrs; 2) be a multidimensional tool evaluating the impact of asthma on physical, emotional and social well-being; 3 ) include an assessment of the young person's concerns about asthma management, particularly medication; 4) take into account possible positive effects of asthma on the adolescent's 
daily life; 5) consider age-related characteristics in cognitive, social and emotional development; 6) be designed for self-administration; 7) be potentially responsive to change over time due to intervention (such as medication, education, behavioural modification).

\section{Methods}

\section{Subjects}

Inclusion criteria. Eligible adolescents were aged 12 17 yrs. They were recruited from three paediatric asthma clinics of two tertiary hospitals. A total of 111 patients with asthma as defined by the American Thoracic Society guidelines [7] were enrolled (demographic data are illustrated in table 1). During the two weeks prior to enrolment, all participants experienced asthma symptoms at least once a week or took asthma medication daily. Participants were excluded if their English knowledge was poor, or if they had other chronic diseases (except atopy) that might affect HRQOL. Ethics approval was obtained from the Royal Children's Research in Human Ethics Committee. Each participant and one of their caregivers gave informed consent.

Sample A. Focus group interviews were held with 14 adolescents (eight females and six males; mean \pm SD age 14.8 $\pm 1.6 \mathrm{yrs}$; duration of asthma $14.1 \pm 1.8 \mathrm{yrs}$ ) as part of the item selection (see below).

Sample B. Item reduction and validation of the final version of the AAQOL, was performed in a single study with 66 adolescents (36 females and 30 males, age $15.5 \pm 1.9 \mathrm{yrs}$, duration of asthma $12.5 \pm 3.0 \mathrm{yrs}$ ).

Sample $C$. To measure the instrument's reproducibility,

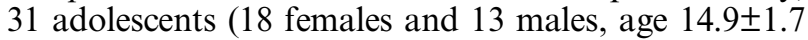

yrs, duration of asthma 11.6. $\pm 2.5 \mathrm{yrs}$ ) completed the final version of the AAQOL twice, two weeks apart. There was no overlap between samples.

\section{Study design}

The questionnaire was developed using a standardized multi-step method [8,9].

Item selection. An initial pool of items was generated by a critical review of the literature including existing HRQOL measures [1, 2, 10, 11], expert opinion and by focus group interviews (sample A). Focus group discussions and three single interviews began with open-ended questions followed by semi-structured interviews. Item generation was discontinued when no new items were identified in two interviews. The selected items were combined to form the preliminary questionnaire.

Item reduction. From the preliminary questionnaire, redundant items with similar meaning and items causing any confusion were eliminated. Applying the "clinical impact" method described by GUYATT and JUNIPER and colleagues [8, 12], the most relevant items were identified. This method is consistent with the definition that quality of life "represents the functional effect of an illness and its consequent therapy upon a patient, as perceived by the patient" [6]. The preliminary questionnaire was given to 66 adolescents (sample B) to identity how frequently the event happened, and how bothered or how important the event was to them. The items which were identified as occurring most frequently and considered most important were selected for the final questionnaire. "Clinical impact" was calculated as the product of the frequency and importance rating of each item. Items were then ranked based on their

Table 1. - Demographic characteristics of the three samples

\begin{tabular}{|c|c|c|c|}
\hline & $\underset{n=14}{\text { Sample A* }}$ & $\begin{array}{c}\text { Sample } B^{* *} \\
n=66\end{array}$ & $\begin{array}{c}\text { Sample } C^{* * *} \\
n=31\end{array}$ \\
\hline \multicolumn{4}{|l|}{ Sex } \\
\hline Male & $6(42.9)$ & $30(45.5)$ & $13(41.9)$ \\
\hline Female & $8(57.1)$ & $36(54.5)$ & $18(58.1)$ \\
\hline Age yrs mean & $14.8 \pm 1.6$ & $15.5 \pm 1.9$ & $14.9 \pm 1.7$ \\
\hline Duration of asthma yrs & $14.1 \pm 1.8$ & $12.5 \pm 3.0$ & $11.6 \pm 2.5$ \\
\hline \multicolumn{4}{|l|}{ Drugs taken } \\
\hline Relieving only & N/A & $9(13.6)$ & $2(6.5)$ \\
\hline Both relieving and preventive & N/A & $57(86.4)$ & $29(93.5)$ \\
\hline \multicolumn{4}{|l|}{ Asthma symptoms } \\
\hline Less than once per week (on daily medication) & $0(0.0)$ & $10(15.2)$ & $3(9.7)$ \\
\hline About once per week & $3(21.4)$ & $22(33.3)$ & $9(29.0)$ \\
\hline About three times per week & $7(50)$ & $16(24.2)$ & $13(41.9)$ \\
\hline About every day & $4(28.6)$ & $18(27.3)$ & $6(19.4)$ \\
\hline Hospitalization during last 12 months because of asthma & N/A & $28(42.4)$ & $8(25.8)$ \\
\hline \multicolumn{4}{|l|}{ Smoking status } \\
\hline Nonsmoker & $13(92.9)$ & $60(90.9)$ & $30(96.8)$ \\
\hline Exsmoker & $0(0.0)$ & $3(4.5)$ & $1(3.2)$ \\
\hline Occasional smoker & $1(7.1)$ & $3(4.5)$ & $0(0.0)$ \\
\hline
\end{tabular}

Data are presented as $\mathrm{n}(\%)$ or mean $\pm \mathrm{SD}$. *: Item selection (focus group interviews); **: item reduction; ***: test-retest reliability. 
"clinical impact" within each domain. Those items which were ranked low and had high ceiling effects (i.e. $>60 \%$ of responses falling into the lowest two categories "hardly any of the time/hardly bothered" and "none of the time/not bothered at all") were dropped first. Of those items showing a Pearson correlation higher than 0.80 , the lower ranked item was omitted. Items were also omitted if they caused a drop in Cronbach's alpha of $>0.10$ (see allocation of items to domains). Within each domain, the items were checked for possible sex differences and age differences to consider the inclusion of items if they were rated as one of the four most relevant items (within the domain) in one subgroup but not the total sample.

Allocation of items to the domains. The domains of the AAQOL were predefined based on; 1) the definition of HRQOL; 2) the World Health Organization's definition of health [13]; 3) the focus group interviews that informed the item selection process; and 4) expert opinion. Based on this, the domains proposed were symptoms, medication, physical activities, emotion and social interaction. In addition, items focussing on positive effects of asthma and asthma-related social support on the adolescent's daily functioning were allocated to a separate domain "positive effects" as their scores are not comparable with the scores of other items. Each item was allocated to one of the predefined domains based on the following criteria; 1) each item's face validity was determined by an expert panel; 2) change in Cronbach alpha of each domain was investigated with the addition or exclusion of items, excluding or allocating the item to one of the other domains if Cronbach alpha dropped $>0.10$; 3) each item was correlated with each domain score to ensure that the item was in the most appropriate scale.

Response scales. For the preliminary as well as the final version of the AAQOL, a seven-point Likert scale was used. The seven-point response scale facilitates interpretation of data as it has been shown that the minimal clinically important difference with this scale is 0.5 [14]. Scores were calculated for each domain, as well as a total score. The total score excluded the "positive effects" domain as the response scores of this domain cannot be meaningfully added to scores of the other domains.

Construct validity. Construct validity was assessed by correlating the scores of the final version with several clinical parameters of asthma severity and lung function and with the scores of three health outcome measures; 1) The PAQLQ [1], an asthma-specific 23item quality of life instrument; 2) The Adolescent Dartmouth Primary Care Cooperative Information Project (COOP) charts (ACOOP) [15], a generic sixitem measure with items covering one of the six domains of physical fitness, emotional feelings, school work, social support, family, health habits; 3) Health status thermometer (HST), a visual analogue scale from $0-100$ with zero being the worst imaginable health status and 100 being equal to perfect health.
All questionnaires were completed by 66 adolescents (sample B). Of these, 45 also completed a 7-day asthma diary to obtain more detailed information on clinical parameters of asthma severity.

It was hypothesized there would be; 1) a high correlation ( $\mathrm{r}=0.7-0.9)$ with the PAQLQ; $)$ a moderate correlation $(\mathrm{r}=0.5-0.7)$ with the ACOOP, 3$)$ a moderate correlation with the HST; 4) a weak correlation $(\mathrm{r}=0.3-0.5)$ with clinical parameters of asthma severity, and 5) weak or no correlation with lung function parameters.

Reproducibility. Thirty-one adolescents (sample C) with stable asthma completed the final version of the AAQOL twice, two weeks apart. The questionnaire was mailed home to participants but completed by telephone to ensure the two week time interval. Participants also answered five screening questions about longitudinal stability of their asthma severity with the goal to distinguish between participants with stable and those with unstable asthma, when calculating intraclass correlation coefficients to measure testretest reliability.

\section{Statistical analysis}

Data analysis was performed using Stata [16]. Pearson correlation coefficients and Spearman rank correlation coefficients were calculated to measure the relationships between outcome measures. To facilitate comparison of results, only Spearman rank correlation coefficients are presented (Pearson correlation coefficients were equal to or slightly higher than these results). Pearson correlation coefficients are reported for interitem and interdomain correlations of the AAQOL.

\section{Results}

\section{Item selection}

The initial pool comprised 89 items. Of these, 13 items were considered redundant and omitted. In addition, three items selected from the literature review were omitted because they were not identified by the focus groups as issues of potential relevance. Therefore, a preliminary questionnaire comprising 73 items was obtained. The majority of the items $(91 \%)$ asked about the negative impact of asthma on HRQOL, and $9 \%$ focused on positive issues in regard to experiencing asthma.

\section{Item reduction}

The product of frequency rating and importance rating for each item was calculated. The items were ranked using this product within each domain. This is illustrated in table 2 for the "physical activities" domain. The reasons for omitting items frorn the 73 item pool of the preliminary questionnaire are listed in table 3 . 
Table 2. - Item ranking for the physical activities domain by impact score

\begin{tabular}{|c|c|c|c|}
\hline Items & $\begin{array}{c}\text { Mean } \\
\text { frequency }\end{array}$ & $\begin{array}{c}\text { Mean } \\
\text { importance }\end{array}$ & Impact** \\
\hline Running made you cough or wheeze* & 3.48 & 4.25 & 17.39 \\
\hline Difficulties participating in long distance sports activities* & 3.71 & 4.26 & 20.29 \\
\hline Have to avoid things that make your asthma symptoms worse* & 4.52 & 4.85 & 25.05 \\
\hline Been restricted in sport, exercising or physical education & 4.59 & 4.76 & 25.80 \\
\hline Been restricted in sports, hobbies or other recreational activities* & 4.68 & 4.91 & 26.80 \\
\hline Been limited in any activities & 4.91 & 4.98 & 28.15 \\
\hline Missed school* & 5.06 & 5.29 & 30.05 \\
\hline Have difficulty when walking uphill or upstairs* & 5.15 & 5.26 & 30.79 \\
\hline Have asthma symptoms because you were laughing & 5.11 & 5.42 & 31.32 \\
\hline Asthma stopped you doing things that you wanted & 5.45 & 5.32 & 32.56 \\
\hline Been restricted in being involved in your hobbies & 5.55 & 5.33 & 32.74 \\
\hline Been limited in going to certain places because they are bad for your asthma & 5.5 & 5.5 & 33.64 \\
\hline How often did asthma affect your school performance & 5.59 & 5.65 & 34.45 \\
\hline Been annoyed by your asthma when dancing & 5.32 & 5.75 & 34.77 \\
\hline Feel restricted in doing household chores & 6.12 & 6.55 & 41.42 \\
\hline Limited fun in sexual activities & 6.85 & 7.4 & 50.95 \\
\hline
\end{tabular}

*: Item included in the final version; **: clinical impact=frequency $\times$ importance (being bothered).

There were no significant sex differences regarding the ranking of the items. However, one item ("feeling bothered by your asthma when walking uphill or upstairs") was rated significantly higher in the older age group (15-17 yrs, item ranking 6) compared with the younger age group (12-14 yrs, item ranking 11). This item was included in the final questionnaire.

\section{Allocation of items}

None of the items allocated to a particular domain by the expert panel showed a significantly higher correlation with the total score of another domain. Of the six items initially grouped as an "environmental" domain, three were ranked low. One item ("experience asthma symptoms because of weather changes and air pollution") was allocated to the "symptom" domain as this item had a correlation of 0.56 with the total of the "symptom" domain, and Cronbach alpha for this domain remained stable $(\alpha=0.85)$ with the allocation of this item. Another item within the "environmental" domain ("have to avoid a situation or place because of cigarette smoke") was allocated to the "social interaction" domain. Although the correlation of this item with the rest of the "social interaction" domain was low $(r=0.26)$, the allocation was considered justified based on the fact that Cronbach alpha of the "social interaction" domain was only reduced by $0.05(\alpha=0.76)$ when including this item. Furthermore on the face of it the item would appear to be related to aspects of social

Table 3. - Reasons for having omitted items

\begin{tabular}{lc}
\hline & $\begin{array}{c}\text { Items } \\
\mathrm{n}(\%)\end{array}$ \\
\hline Low ranking/high ceiling effects & $33(80)$ \\
Ambiguous wording & $2(5)$ \\
Correlation $>0.80$ with higher ranked item & $3(7)$ \\
Similar wording & $2(5)$ \\
Did not fit into any domain & $1(2)$ \\
Total number of items omitted & 41 \\
\hline
\end{tabular}

interaction, since either smoking colleagues, friends, relatives etc. have to be avoided (direct impact on social interaction), or places where social interaction commonly occur (pubs, restaurants etc.) need to be avoided because of smokers not known to the person. This item may therefore simply measure a different aspect of social interaction than do the rest of the items in the "social interaction" domain.

Internal consistency was high for all domains as well as the questionnaire's total score (table 4). Pearson correlations among the domains ranged from 0.68 0.78 , and the domains correlated with the total score of the AAQOL between 0.86 and 0.92 .

\section{Construct validity}

Data on construct validity are presented in table 5 . The AAQOL had a high correlation $(\rho=0.81)$ with the PAQLQ as expected. Even excluding items common to both scales, the correlation remained high $(\rho=0.79)$. There was a moderate correlation with the ACOOP $(\rho=-0.60$; negative correlated because of inverse scoring) and a weak but significant correlation $(\rho=0.46)$ with the HST. Both the AAQOL and the PAQLQ correlated weakly to moderately (but statistically significantly) with patient-rated symptom severity, number of hospitalizations during the last 12 months, severity of coughing and severity of wheezing. No statistically significant correlation was found with the

Table 4. - Cronbach alpha for each domain individually and Adolescent Asthma Quality of Life Questionnaire (AAQOL) total score

\begin{tabular}{ll}
\hline Symptoms & 0.85 \\
Medication & 0.78 \\
Physical activities & 0.85 \\
Emotion & 0.90 \\
Social interaction & 0.76 \\
Positive effects & 0.70 \\
AAQOL* & 0.93 \\
\hline
\end{tabular}

\footnotetext{
*: Not including the domain "positive effects".
} 
Table 5. - Spearman rank correlations between different health outcome measures and with asthma severity parameters

\begin{tabular}{|c|c|c|c|c|}
\hline & AAQOL $^{\#}$ & PAQLQ & ACOOP & HST \\
\hline PAQLQ & $0.81 * * *$ & & & \\
\hline ACOOP & $-0.60 * * *$ & $-0.62 * * *$ & & \\
\hline HST & $0.46^{* * *}$ & $0.64 * * *$ & $-0.36^{*}$ & \\
\hline Patient-rated symptom severity & $-0.45^{* * *}$ & $-0.49 * * *$ & $0.40 * * *$ & $-0.33^{*}$ \\
\hline No. of hospitalizations in last 12 months & $-0.51 * * *$ & $-0.48 * * *$ & $0.30^{*}$ & $-0.32 *$ \\
\hline No. of medications per day & -0.25 & $-0.33 *$ & $0.36^{*}$ & -0.19 \\
\hline Coughing last 7 days (asthma diary) & $-0.52 * * *$ & $-0.60 * * *$ & 0.30 & $-0.61 * * *$ \\
\hline Wheezing last 7 days (asthma diary) & $-0.65^{* * *}$ & $-0.57 * * *$ & $0.39 *$ & $-0.51 * * *$ \\
\hline Sleeping last 7 days (asthma diary) & -0.29 & $-0.60 * * *$ & $0.31 *$ & $-0.55^{* * *}$ \\
\hline FEV1 & 0.15 & 0.24 & -0.13 & 0.11 \\
\hline
\end{tabular}

\#: Total score without the "positive effects" domain; AAQOL: Adolescent Asthma Quality of Life Questionnaire; PAQLQ: Pediatric Asthma Quality of Life Questionnaire; ACOOP: Adolescent Dartmouth Primary Care Cooperative Information Project Charts; HST: Health Status Thermometer; FEV1: forced expired volume in one second; *: correlation statistically significant at $\mathrm{p}<0.05 ; * * *$ : correlation statistically significant at $\mathrm{p}<0.001$.

number of medications taken, and neither the AAQOL nor the PAQLQ showed a statistically significant correlation with forced expired volume in one second (FEV1) (\% predicted). The HST correlated moderately and equally as highly as the AAQOL and the PAQLQ with coughing and wheezing but less with other asthma severity parameters when compared to the AAQOL and the PAQLQ.

\section{Reproducibility}

Thirty-one adolescents participated in the test-retest reliability phase of questionnaire development (sample C), by completing the final version of the AAQOL twice, two weeks apart. Using data of participants with stable asthma only, agreement between subscale and total scores for the first and the second completion of the AAQOL was calculated using intraclass correlation coefficients. The items of the symptom domain were shown to be more sensitive in defining stable asthma (due to seven-point Likert scale) than the five screening questions asked with each interview (threepoint Likert scale only). To calculate reproducibility of all domains except the symptom domain, 22 participants with changes of the symptom domain subscore within two standard deviations of the mean were defined as stable. To measure reproducibility of the symptom domain, 20 participants who answered three out of five screening questions as "being stable" were considered to have stable asthma.

Test-retest reliability was high for all domains as well as the total score, with intraclass correlation coefficients ranging from 0.76 for the symptom domain to 0.85 for the emotion domain and 0.90 for the total score. Thus, the domain scores as well as the total score are highly reproducible.

\section{Discussion}

The aim of this study was to develop and validate an asthma-specific quality of life questionnaire for adolescents with asthma. The final version of the AAQOL contains 32 items covering six domains of HRQOL. It is designed for self-administration with most respon- dents requiring 5-7 min for completion. The AAQOL showed good construct validity given the correlations with other quality of life measures as anticipated. The high test-retest reliability provides the basis for good responsiveness of the AAQOL.

As HRQOL may be influenced by the individual's current stage of cognitive, social and emotional development, it has been argued that HRQOL in adolescents needs to be addressed separately [17]. The AAQOL takes into account the key developmental aspects of adolescence as it was specifically designed for the age range $12-17$ yrs. The AAQOL is self-completed and focuses on the adolescent's subjective perception. Age appropriateness is ensured by including items which were defined as particularly important by this age group. The disadvantage of this adolescent-specific approach is that it does not allow comparison of data between children and adolescents. However, the minimal age required for self-reported HRQOL assessment has not yet been determined. In contrast to generic HRQOL instruments, disease-specific questionnaires require the cognitive capability of respondents to not only recognize that they feel limited in certain domains of their daily functioning, but to answer the more difficult question as to whether they perceive these limitations are caused by chronic disease (e.g. asthma). Further research is required to investigate whether children $<12$ yrs old who have not generally achieved formal operational thinking, are sufficiently capable to answer disease-specific HRQOL instruments.

Item reduction was performed using the "clinical impact" method which, in contrast to the more traditional factor analysis, primarily considers the clinical relevance of the selected items, as perceived by the target population itself. The "clinical impact" method has been increasingly used recently $[1,12,18$, 19], as it is consistent with most definitions of HRQOL which emphasize the need to focus on the target individual's personal perception. HRQOL questionnaires developed using the "clinical impact" method focus on the target individual's perception when answering the items, but also ensure that the items included in the questionnaire are those which are perceived to be clinically relevant by the target population itself. In contrast, factor analysis does not take into account the perception of clinical relevance of 
items by the target population. Instead, item reduction is performed based solely on mathematical linkage between items. However, there are two potential disadvantages of the "clinical impact" method. Firstly, to the authors' knowledge there is currently no statistical method supporting sample size estimations. Secondly, the "clinical impact" method does not assist in defining the domains to be covered by the instrument. While this conforms with the aim of primarily focussing on the target group's perception of issues relevant to them, this approach might lead to the development of an unbalanced instrumeut or lead to the definition of domains that are difficult to interpret. The domains were therefore predefined as outlined in the methods section. The "clinical impact" method was then applied within each domain to reduce the number of items.

Some items in the AAQOL focus on positive impacts that asthma might have on the individual's daily life. This "positive effects" domain is unique as none of the currently available asthma-specific HRQOL instruments have considered positive effects of disease on HRQOL. It contrasts with the assumption that chronic illness and disability is only associated with negative impacts. The difficulty with the "positive effects" domain was how to assess its construct validity which means testing hypotheses of how the domain would correlate with other variables. When considering the content of each item (e.g. "how often has your asthma brought you closer as a family?"), it was not possible to make hypotheses of how the items would correlate with other HRQOL instruments or clinical parameters of asthma severity. However, as the items of the "positive effects" domain were rated highly important by the adolescents, it was decided to include the "positive effects" domain in the AAQOL despite our lack of measurement of construct validity. Content validity of the "positive effects" domain was rated fairly high by the expert panel and Cronbach alpha was good with $\alpha=0.70$. It may be possible to use the AAQOL without this domain, as the "positive effects" domain does not contribute to the AAQOL's total score. However, as the AAQOL's breadth of HRQOL assessment may be reduced, this approach is not currently recommended.

Regarding the calculation of the total score, it was decided not to weight individual domain scores differently. In the final version all the domains contained either five or six items and there is evidence, that under these circumstances, weighting subscores may significantly complicate data analysis without enhancing the accuracy of HRQOL assessment [20]. Also, in a pilot study assessing the potential relevance of different domains in adolescents with asthma, all domains were rated equally important (except the "positive effects" domain which was not investigated) [21].

The AAQOL total score showed a high correlation with the PAQLQ and a moderate correlation with the ACOOP as expected, providing good evidence for the AAQOL's construct validity. In comparison with the PAQLQ, the AAQOL more specifically addresses issues of particular importance to adolescents. Furthermore, the AAQOL provides a more comprehensive HRQOL assessment by focussing on a broader range of domains than the PAQLQ. The AAQOL therefore provides a more age-appropriate and descriptive picture of HRQOL of adolescents with asthma with the potential to facilitate a better understanding of the burden of illness and its consequent treatment for this population.

The AAQOL showed a moderate correlation with the majority of parameters of clinical asthma severity investigated in this study. As anticipated, no significant correlation was found with lung function parameters. This finding is similar to other asthma-specific HRQOL measures. The lack of significant correlation with lung function parameters is not interpreted as reduced validity, but rather highlights the necessity of combining physiologic measures with HRQOL instruments to complement health outcome measurements.

The AAQOL has not been validated for use in adolescents with infrequent episodic asthma, but only for those with regular asthma symptoms. While infrequent episodic asthma may also impact upon the adolescent's daily functioning, the impact may be different from that of frequent episodic or persistent asthma. For example, the adolescent with infrequent episodic asthma may be threatened by asthma attacks but may be less likely to be significantly limited in physical activities and social interaction. Further studies are required to assess the value of asthmaspecific HRQOL instruments in young people with infrequent episodic asthma.

A limitation of this study is the current lack of measurement of the responsiveness to change over time due to intervention. However, a basic requirement of responsiveness is the instrument's reproducibility (testretest reliability as a measure of longitudinal stability) which has been shown to be excellent for the AAQOL. The validation of HRQOL instruments such as the AAQOL is an ongoing process with each clinical study providing further data on the AAQOL's validity.

In summary, the Adolescent Asthma Quality of Life Questionnaire is an age-appropriate and valid asthmaspecific health-related quality of life questionnaire for use in adolescents with asthma. It more comprehensively measures a range of domains in adolescents with asthma than any of the currently available asthmaspecific quality of life instruments. As with all instruments currently available for use in children and adolescents with asthma, further research is encouraged to confirm the Adolescent Asthma Quality of Life Questionnaire's usefulness and validity as a comprehensive health outcome measure in adolescents.

\section{References}

1. Juniper EF, Guyatt GH, Feeny DH, Ferrie PJ, Griffith LE, Townsend M. Measuring quality of life in children with asthma. Qual Life Res 1996; 5: 35-46.

2. French DJ, Christle MJ, Sowden AJ. The reproducibility of the childhood asthma questionnaires: measures of quality of life for children with asthma aged 4-16 years. Qual Life Res 1994; 3: 215-224.

3. Creer TL, Wigal JK, Kotses H, Hatala JC, McConnaughy $\mathrm{K}$, Winder JA. A life activities questionnaire for childhood asthma. JAsthma 1993; 30: 467-473.

4. Usherwood TP, Scrimgeour A, Barber JH. Questionnaire to measure perceived symptoms and disability in asthma. Arch Dis Child 1990; 65: 779-781. 
5. Rutishauser C, Sawyer SM, Bowes G. Quality-of-life assessment in children and adolescents with asthma. Eur Respir J 1998; 12: 486-494.

6. Schipper H, Clinch J, Powell V. Definitions and conceptual issues. In: Spilker B, ed. Quality of life assessments in clinical trials. New York, Raven Press, 1990; 11-35.

7. National Asthma Education and Prevention Program. Expert Panel Report: Guidelines for the Diagnosis and Management of Asthma. National Institutes of Health pub no 91-3642. Bethesda, MD, 1991.

8. Guyatt GH, Bombardier C, Tugwell PX. Measuring disease-specific quality of life in clinical trials. $C M A J$ 1986; 134: 889-895.

9. Juniper EF, Guyatt GH, Jaeschke R. How to develop and validate a new health-related quality of life instrument. In: Spilker B, ed. Quality of Life and Pharmacoeconomics in Clinical Trials. Philadelphia, Lippincott-Raven Publishers, 1996; pp. 49-56.

10. Juniper EF, Guyatt GH, Epstein RS, Ferrie PJ, Jaeschke R, Hiller TK. Evaluation of impairment of health related quality of life in asthma: development of a questionnaire for use in clinical trials. Thorax 1992; 47: 76-83

11. Marks GB, Dunn SM, Woolcock AJ. A scale for the measurement of quality of life in adults with asthma. $J$ Clin Epidemiol 1992; 45: 461-472.

12. Juniper EF, Guyatt GH, Streiner DL, King DR. Clinical impact versus factor analysis for quality of life questionnaire construction. J Clin Epidemiol 1997; 50: 233-238.
13. World Health Organization. Constitution of the World Health Organization. Geneva, WHO, 1947.

14. Jaeschke R, Singer J, Guyatt GH. Measurement of health status: ascertaining the minimal clinically important difference. Control Clin Trials 1989; 10: 407-415.

15. Wasson JH, Kairys SW, Nelson EC, Kalishman N, Baribeau P. A short survey for assessing health and social problems of adolescents. J Fam Pract 1994; 38: 489-494.

16. StataCorp. 1997. Stata Statistical Software: Release 5.0, College Station, Texas: Stata Corporation.

17. Richards JM, Hemstrect MP. Measures of life quality, role performance, and functional status in asthma research. Am J Respir Crit Care Med 1994; 149: S31S39.

18. Parkin PC, Kirpalani HM, Rosenbaum PL, et al. Development of a health-related quality of life instrument for use in children with spina bifida. Qual Life Res 1997; 6: 123-132.

19. Juniper EF, Guyatt GH, Epstein RS, Ferrie PJ, Jaeschke R, Hiller TK. Evaluation of impairment of health-related quality of life in asthma: development of a questionnaire for use in clinical trials. Thorax 1992; 47: 76-83.

20. Streiner DL, Norman GR. Health measurement scales: a practical guide to their development and use. Oxford, Oxford University Press 1995; 85-103.

21. Rutishauser C, Sawyer S, Bowes G. Quality of life questionnaires in adolescents with asthma: a pilot study, Annual Scientific Meeting, TSANZ. Adelaide, 1998. 